\section{Dispenser printing of electrochromic display on textiles for creative applications}

\author{
Y. Wei ${ }^{\bowtie}$, X. Wang, R. Torah and J. Tudor
}

For the first time, the design, fabrication and testing of a dispenserprinted electrochromic (EC) display on fabric using Poly(3,4-ethylenedioxythiophene)-poly(styrenesulfonate) (PEDOT:PSS) as the EC material are reported. The entire display is based on a lateral structura design in which the electrodes are located adjacent to each other in the same plane. The display is directly printed onto a, polyvinyl chloride (PVC)-coated, 100\% polyester woven fabric which is commonly used in the creative industries. Each layer was cured below $130^{\circ} \mathrm{C}$ to ensure no thermal damage to the fabric. Each display pixel, consisting of the colour changing and counter electrodes, was separately driven at two voltage direct currents (VDCs). The colour change between pale blue and dark blue was controlled by switching the polarity using a microcontroller. Two demonstrators, a $3 \times 3$ pixel matrix display and a seven segment display, were achieved with an average switching speed of $5 \mathrm{~s}$

Introduction: The electrochromic (EC) effect occurs in materials which change their optical properties in response to an applied electric potential. When the potential stops or reverses, these materials return to their original optical state in a reversible way [1]. The colour change is commonly between a transparent ('bleached') state and a coloured state or between two coloured states. EC displays have attracted much interest in both academia and industry resulting in a global market of \$2 million in 2016 [2]. EC displays are commonly fabricated on rigid substrates (e.g. glass) [3] but have also been demonstrated on plastic/paper substrates $[4,5]$. EC devices on textiles have been reported $[6,7]$ but these devices are only considered to be an indicator because only one colour on one area is displayed at once, thereby limiting amount of information which can be displayed. In addition, the prior fabrication of EC devices on textiles was either by soaking [6] where entire fabric is immersed, and therefore functionalised or separately fabricated on a plastic film and then attached to the textile [8].

The EC display reported in this Letter is fabricated using a bespoke dispenser printer where the electronic inks are directly printed on the fabric in any geometric layout and only where they are needed without the use of masks, screens or other tooling. This allows rapid prototyping, minimises resource usage and maximises fabric breathability. Poly(3,4-ethylenedioxythiophene)-poly(styrenesulfonate) (PEDOT:PSS) is used as the EC material as it is capable of changing its colour from pale blue to dark blue under an applied potential. PEDOT:PSS has also been proved to be suitable for a flexible EC display $[5,9,10]$ and its curing temperature of $<130^{\circ} \mathrm{C}$ causes no damage to the fabric.

Table 1: List of inks used for dispenser-printed EC display

\begin{tabular}{|c|c|}
\hline Layer & Material \\
\hline Electrode/EC & Fabinks TC-C4005 (PEDOT:PSS) \\
\hline Busbar & Fabinks TC-C4007 (silver based) \\
\hline Electrolyte & lithium ion based \\
\hline Side wall & UV curable dielectric ink EFV4 \\
\hline Masking & UV curable opaque white SPF6 \\
\hline
\end{tabular}

Material selection: A polyvinyl chloride (PVC)-coated $100 \%$ polyester woven fabric, supplied by Berger Textil Germany, was used as the substrate because this fabric is one of the most common fabrics used in the creative industries and is the base material for inkjet-printed display banners, exhibition stands and window blinds [11]. Fabinks TC-C4005 PEDOT:PSS ink was used as the EC material [12]. Fabinks TC-C4007 is used as the conductor for the busbar/connection pads, since it is compatible with dispenser printing and the fabric.

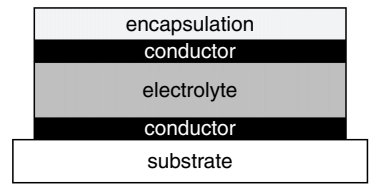

a $b$

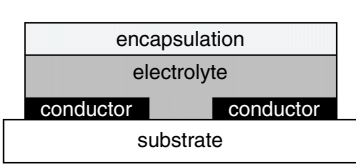

Fig. 1 Basic structure of EC device

$a$ Vertical

$b$ Lateral arrangement
Table 1 shows the ink of each layer used for the fabrication. The PEDOT:PSS, silver and electrolyte inks are thermally curable at 125,125 and $50^{\circ} \mathrm{C}$, respectively. The ultraviolet (UV) curable inks (side wall and masking layer), from Electra Polymers Ltd., are cured, after printing, using a Panacol-Elosol UV-P 280 UV point source. The printed layers were exposed to $2000 \mathrm{~mW} / \mathrm{cm}^{2}$ radiation for $60 \mathrm{~s}$.

Display structure: The EC display can be configured based on the electrode arrangement as shown in Fig. 1.

The vertical structure consists of an electrolyte layer sandwiched between two electrodes, whereas the lateral structure consists of an electrolyte layer on top of two adjacent electrodes which are in the same plane. The switching response is faster in the vertical structure due to a shorter migration path for ions through the electrolyte but the electrolyte has to be opaque, so that only the colour change of the top electrode is visible and not that of the bottom electrode. In this research, the lateral structure is selected as being the most suitable for dispenser printing.

Fabrication and testing: The dispenser printer is a bespoke machine developed at the University of Southampton. The material is deposited via a pressurised syringe onto the substrate which is controlled in three dimensions using an XYZ stage system according to the desired printed pattern. The printing resolution is dependent on the accuracy of the moving stages, the size of the syringe nozzle and the material rheology. The amount of ink deposited is controlled by the pressure acting on the syringe and the printing mode used (i.e. droplet or continuous printing). Each layer of the EC display was dispenser printed using the parameters as shown in Table 2 .

Table 2: Dispenser printing parameters

\begin{tabular}{|c|c|c|c|c|}
\hline Ink & $\begin{array}{c}\text { Printing } \\
\text { mode }\end{array}$ & $\begin{array}{c}\text { Dispensing time } \\
(\mathrm{ms})\end{array}$ & $\begin{array}{c}\text { Pressure } \\
(\mathrm{kPa})\end{array}$ & $\begin{array}{c}\text { Vacuum } \\
(\mathrm{kPa})\end{array}$ \\
\hline $\begin{array}{c}\text { PEDOT: } \\
\text { PSS }\end{array}$ & droplet & 13 & 27 & 0.5 \\
\hline Silver & droplet & 33 & 45 & 1.5 \\
\hline Electrolyte & continuous & N/A & 20 & 0.1 \\
\hline EFV4 & continuous & N/A & 25 & 0.8 \\
\hline SPF6 & continuous & N/A & 10 & 1.5 \\
\hline
\end{tabular}
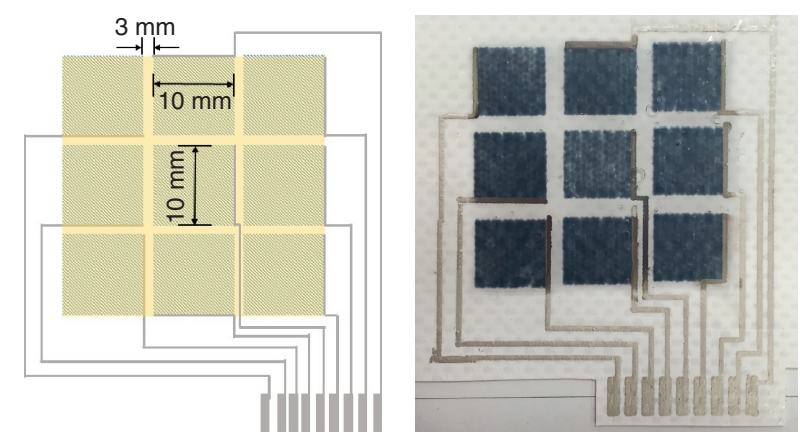

Fig. $23 \times 3$ matrix display: (left) design layout and (right) printed sample

Pixel matrix display: In this demonstrator, the $3 \times 3$ matrix display consists of nine $10 \mathrm{~mm} \times 10 \mathrm{~mm}$ PEDOT:PSS electrodes adjacent and in the same plane and with a $3 \mathrm{~mm}$ gap between every two electrodes. Here, $3 \mathrm{~mm}$ is selected as a compromise distance because it leaves enough space for the electrical connection from the centre electrode and also enables the colour change of each electrode within $5 \mathrm{~s}$. The design layout and printed sample are as shown in Fig. 2. The pitch between the connection pads on the bottom right in Fig. 2 is $2.54 \mathrm{~mm}$ to be compatible with commercial connectors. The electrolyte was printed over the entire area of the nine electrodes and then protected by a transparent polyester adhesive film of $75 \mu \mathrm{m}$ thickness. Each pixel was individually controlled using a microcontroller and resistor bridge which provides a stable two voltage direct currents (VDCs) which ensures a reversible colour change of the PEDOT:PSS.

Fig. 3 shows examples of printed EC matrix display with pixels activated. Each pixel was able to change between pale blue and dark blue around $5 \mathrm{~s}$. 

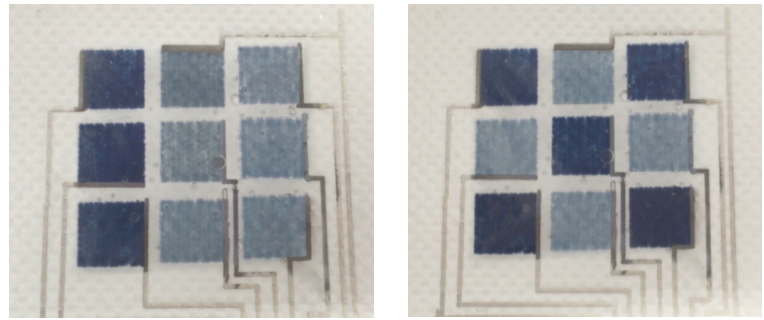

Fig. 3 Examples of EC display with pixels activated

Seven segment display: As a second demonstrator, a seven segment display was achieved using the structure as shown in Fig. 4.

\begin{tabular}{|c|c|c|c|}
\hline & & \multicolumn{2}{|l|}{ mask } \\
\hline \multicolumn{4}{|c|}{ encapsulation } \\
\hline & \multicolumn{2}{|c|}{ electrolyte } & \\
\hline $\mathrm{Ag}$ & PEDOT & PEDOT & $\mathrm{Ag}$ \\
\hline & & & \\
\hline
\end{tabular}

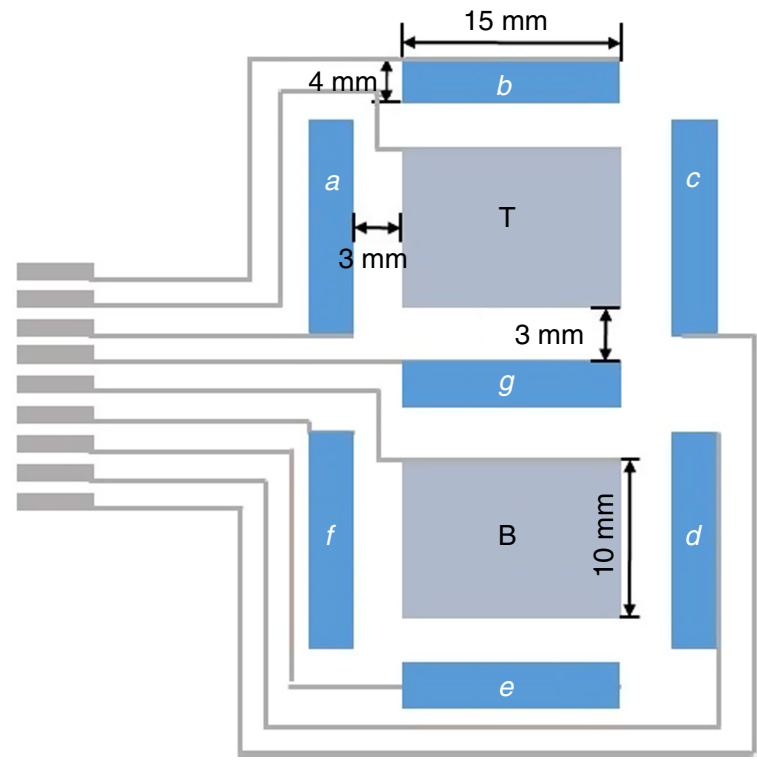

Fig. 4 Cross-section and top drawings of seven segment EC display
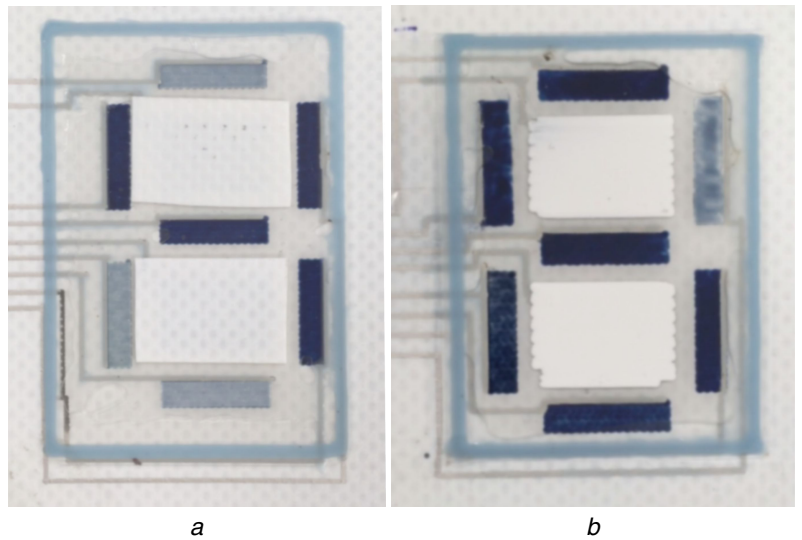

Fig. 5 Photographs of numbers displayed on EC display

$a$ Four

$b$ Six

In the seven segment design, segments $\mathrm{a}, \mathrm{b}$ and $\mathrm{c}$ share the counter electrode $\mathrm{T}$, whereas segments $\mathrm{d}$, e, f and $\mathrm{g}$ share the counter electrode B. Each segment was separately connected to a resistor bridge, and then a microcontroller, which allows the number to be displayed based on the control signals provided by a PC. Fig. 5 shows examples of numbers activated on the display and each number is able to be displayed within a $5 \mathrm{~s}$ timescale.

Conclusions: This research demonstrates dispenser-printed displays on fabric using PEDOT:PSS as the EC material. The use of dispenser printing allows the design to be modified without needing extra tooling such as the screens which are required for each screen printing layer and design. The PEDOT:PSS changes from pale blue to dark blue under the application of two VDCs. The colour change is reversible by changing the polarity of the supply potential which is controlled using a microcontroller. For both the matrix and the seven segment display, the colour of printed PEDOT:PSS electrode changes its colour in $\sim 5 \mathrm{~s}$. For both displays, the pixel and segment sizes as well as the electrode separation can be further reduced by using a finer nozzle to allow a more detailed monochrome display to be achieved.

(C) The Institution of Engineering and Technology 2017

Submitted: 11 January 2017

doi: $10.1049 / \mathrm{el} .2017 .0119$

One or more of the Figures in this Letter are available in colour online.

Y. Wei, X. Wang, R. Torah and J. Tudor (School of Electronics and Computer Science, University of Southampton, Southampton SO17 1BJ, United Kingdom)

凶-mail: y.wei@soton.ac.uk

\section{References}

1 Mattila, H.: 'Intelligent textiles and clothing' (Woodhead Publishing Limited and CRC Press LLC, Cambridge, UK, 2006)

2 Das, R., and Harrop, P.: 'Printed, organic and flexible electronics forecasts, players and opportunities 2016-2026', IDTechEx Market Data, 2016

3 Pettersson, H., Gruszecki, T., Johansson, L., Edwards, M., Hagfeldt, A., and Matuszczyk, T.: 'Direct driven electrochromic displays based on nanocrystalline electrodes', Displays, 2004, 25, pp. 223-230

4 Ding, Y., Invernale, M., Mamangun, D., Kumar, A., and Sotzing, G.: 'A simple low waste and versatile procedure to make polymer electrochromic devices', J. Mater. Chem., 2011, 21, pp. 11873-11878

5 Tehrani, P., Hennerdal, L., Dyer, A., Reynolds, J., and Berggren, M. 'Improving the contrast of all printed electrochromic polymer on paper display', J. Mater. Chem., 2009, 19, pp. 1799-1802

6 Invernale, M., Ding, Y., and Sotzing, G.: 'All organic electrochromic spandex', Appl. Mater. Interfaces, 2010, 2, (1), pp. 296-300

7 Ding, Y., Invernale, M., and Sotzing, G.: 'Conductivity trends of PEDOT:PSS impregnated fabric and the effect of conductivity on electrochromic textile', Appl. Mater. Interfaces, 2010, 2, (6), pp. 1588-1593

8 Meunier, L., Kelly, F., Cochrane, C., and Koncar, V.: 'Flexible displays for smart clothing: part II-electrochromic displays', Indian J. Fibre Text. Res., 2011, 36, pp. 429-435

9 Andersson, P., Forchheimer, R., Tehrani, P., and Berggren, M.: 'Printable all organic electrochromic active-matrix display', $A d v$. Funct. Mater., 2007, 127, pp. 3074-3082

10 Tehrani, P., Isakim, J., Mammo, W., Andersson, M., Robinson, N., and Berggren, M.: 'Evaluation of active materials designed for use in printable electrochromic polymer displays', Thin Solid Films, 2006, 515, pp. 2485-2492

11 Datasheet Back-lighttex, Berger Textil

12 'Fabinks Ltd.,'. Available at http://www.fabinks.com 